\title{
Modélisation stochastique de la croissance et du développement du système racinaire de jeunes pêchers. I. Estimation et validation du modèle
}

\author{
L Pagès 1 , J Chadœuf 2 , J Kervella 3 \\ INRA, ${ }^{1}$ Laboratoire d'agronomie; \\ 2 Laboratoire de biométrie; \\ ${ }^{3}$ Station de recherches fruitières méditerranéennes, Domaine St-Paul, BP 91, 84143 Montfavet Cedex, France
}

(Reçu le 25 novembre 1991; accepté le 26 février 1992)

\begin{abstract}
Résumé - Nous avons étudié le développement racinaire de jeunes semis de pêcher (Prunus persica variété GF 305). Pour ce faire, 45 arbres ont été cultivés pendant 6 semaines sur perlite. Tous les 5 jours, 5 plants ont été dépotés. La longueur et la distance à la base de l'axe qui le porte ont été mesurées pour chacun de leurs axes racinaires. La croissance de chaque axe a été modélisée par une fonction puissance de son âge. Les courbes de croissance d'un même ordre de ramification sont affines. Le coefficient d'affinité dépend de ce que nous avons appelé la "vigueur" de la racine. Le processus de ramification a été modélisé par un processus de Poisson temporel à paramètre aléatoire (mixed Poisson). L'étude statistique du modèle montre une variabilité importante de la vigueur pour les axes d'ordre 2, 3 et 4. De l'ordre 1 vers l'ordre 4, la croissance évolue progressivement du type indéfini vers le type défini, et la vitesse de croissance diminue. Parallèlement, l'intensité du processus de ramification diminue. Ce modèle nous permet de décrire en moyenne et en variabilité les cinétiques de variables globales comme la longueur totale ou le nombre de racines d'un ordre donné. Il nous a aussi permis d'évaluer la sensibilité de ce type de modèle au choix des lois ou distributions des variables aléatoires utilisées pour décrire la variabilité observée.
\end{abstract}

système racinaire $/$ Prunus persica $=$ pêcher $/$ croissance $/$ architecture $/$ modèle stochastique

Summary - Stochastic modeling of growth and development of the root system of young peach tree seedlings. I. Estimation and validation of the model. The growth and branching of the root system of young peach tree seedlings (Prunus persica cultivar GF 305) have been studied. Forty-five seedlings were grown on perlite during 6 weeks. Five plants were dug out every 5 days and their root axes length and position measured. The growth of each axis has been modeled using a power function depending on its age. For a given branching order, the growth curves of all the axes are proportional. The proportionality coefficient depends on a root growth potential ("vigor"). The branching process has been modeled using a temporal Poisson point process with random parameter (mixed Poisson). The estimation procedure has shown a great variability of the root growth potential for the branching orders number 2, 3 and 4. From order 1 to order 4, root growth evolved from an indefinite type to a definite type and the growth rate decreased. Similarly, the intensity of the branching process decreased from order 1 to order 3. Our model allowed us to describe the mean and variability of the kinetics of global state variables such as total root length and total root number of a given branching order. It also allowed us to analyse the sensitivity of such a model to the choice of laws of the random variables used to describe the observed variabilities.

root system $/$ Prunus persica $=$ peach tree $/$ growth $/$ architecture $/$ stochastic model 


\section{INTRODUCTION}

La compréhension de la fonction d'absorption d'un système racinaire in situ nécessite une meilleure connaissance de sa croissance, de son développement, et de son architecture. Dans de nombreuses situations au champ en effet, ce sont les résistances au niveau des transferts dans le sol jusqu'aux racines qui limitent l'absorption d'eau et d'éléments minéraux (De Willingen et Van Noordwijk, 1987; Habib et al, 1988; Passioura, 1988). Le niveau maximal de l'absorption est donc en partie déterminé par la configuration spatiale du système racinaire et de son évolution.

Pour étudier cette configuration spatiale de façon dynamique, la modélisation est un outil privilégié (Diggle, 1988; Pagès et Aries, 1988; Pagès et al, 1989). Comme le montrent ces travaux, la modélisation permet en effet, à partir d'une synthèse des connaissances concernant la morphogénèse racinaire, de simuler au cours du temps l'architecture tridimensionnelle et, à partir de là, la configuration spatiale de systèmes racinaires.

Pour décrire la croissance et le développement du système racinaire au cours du temps, différents modèles ont été proposés (Hackett et Rose, 1972; Lungley, 1973; Rose, 1983; Porter et al, 1986). Ces modèles attribuent de façon déterministe des vitesses de croissance et de ramification pour chacun des ordres de ramification. Ces vitesses sont des valeurs moyennes estimées pour les conditions de culture étudiées.

Pourtant, même dans des conditions de culture très homogènes, il a été montré une grande variabilité dans les caractéristiques de croissance et de ramification pour les racines d'un ordre donné chez différentes espèces : pois (Yorke et Sagar, 1970), maïs (Jordan, 1986), chênes et acacias (Colin-Belgrand et al, 1989), pêcher (Pagès et al, 1991). Cette variabilité peut être une composante importante pour le fonctionnement d'un système racinaire en tant que capteur. Ainsi, la variabilité dans la croissance des racines, par exemple, peut conférer au système racinaire une plus grande extension d'une part, et une meilleure répartition dans l'espace colonisé d'autre part.

Nous nous proposons donc dans cet article de contribuer aux travaux de modélisation de la mise en place du système racinaire par la présentation, l'estimation et la validation d'un modèle stochastique. Nous présenterons l'expérimentation dont sont issues les données utilisées pour l'estimation des paramètres ainsi que les hypothèses du modèle. Nous discuterons ensuite l'intérêt du modèle pour décrire les cinétiques de croissance et de ramification observées, aussi bien en moyenne qu'en variabilité. Ce travail porte sur le système racinaire de jeunes pêchers, mais la démarche est plus générale et sans doute applicable à d'autres espèces.

\section{EXPÉRIMENTATION}

L'expérimentation a été réalisée sur la variété GF 305, lignée pure de pêcher (Prunus persica L Batsch).

Les noyaux sont cassés et les amandes sont trempées pendant $24 \mathrm{~h}$ dans une solution aqueuse de 6-benzyl-amino-purine à $200 \mathrm{mg} / \mathrm{l}$ pour lever leur dormance (Rouskas et al, 1980). Ces amandes sont ensuite débarrassées de leurs téguments pour homogénéiser la durée avant émergence de la plantule, puis elles sont désinfectées par trempage pendant 15 min dans une solution d'hypochlorite de calcium à $80 \mathrm{~g} / /$, de façon à limiter les attaques fongiques.

Les amandes ainsi traitées sont mises à germer directement en place, à raison de 2 amandes par pot. Dès le début de croissance des plantes (repéré par l'apparition de la tigelle entre les 2 cotylédons qui s'écartent), on procède au "démariage" pour ne laisser dans chaque pot que la plante la mieux développée.

Les jeunes arbres sont cultivés dans des conteneurs cylindriques de $15 \mathrm{I}(30 \mathrm{~cm}$ de profondeur et $25 \mathrm{~cm}$ de diamètre) remplis de perlite expansée et placés en chambre de culture. La température est de $25 \pm 1{ }^{\circ} \mathrm{C}$, l'hygrométrie de $80 \pm 10 \%$. L'éclairement est d'environ 250 $\mu \mathrm{mol} . \mathrm{m}^{-2} . \mathrm{s}^{-1}$, avec $15 \mathrm{~h}$ de jour et $9 \mathrm{~h}$ de nuit. Les plants sont arrosés quotidiennement jusqu'à drainage du substrat, avec de l'eau permutée pendant les 5 premiers jours puis avec de la solution Hoagland à demi-concentration en alternance ( 1 jour sur 2) avec de l'eau permutée.

Nous avons ainsi cultivé 45 arbres au total. L'expérimentation a duré 6 semaines. Les observations sur le développement du système racinaire ont été réalisées à 9 dates réparties tout au long de cette période.

À chaque date, nous avons prélevé un lot de 5 arbres tirés au hasard. Leurs systèmes racinaires ont été soigneusement débarrassés du substrat par un lavage doux. Pour chacun des axes (ou racines) des systèmes racinaires, nous avons noté (fig 1): 


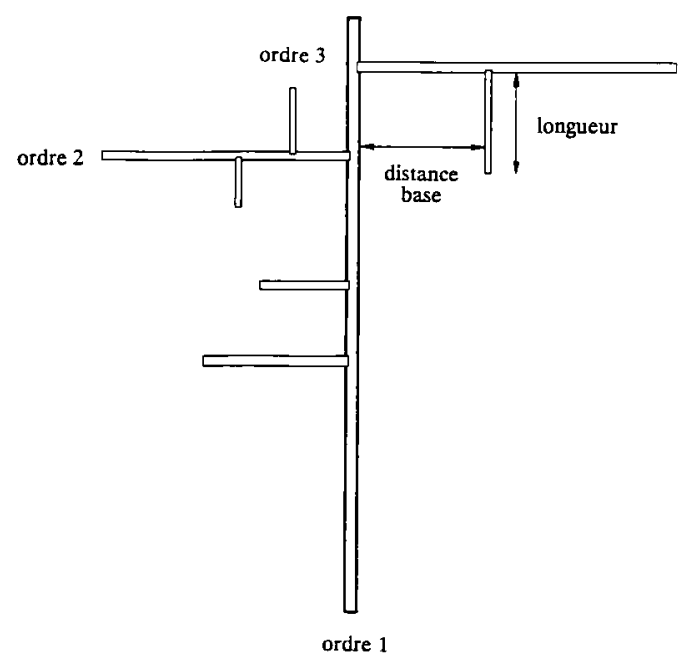

Fig 1. Schéma décrivant les mesures effectuées.

- son numéro d'identification;

- son ordre (l'ordre 1 est le pivot, directement issu de la graine; l'ordre $\mathrm{n}+1$ est porté par l'ordre $\mathrm{n}$ ),

- sa longueur,

- le numéro d'identification de la racine qui le porte,

- la distance de son point d'insertion à la base de cette racine.

Ces mesures ont été faites de façon exhaustive sur tous les axes de tous les arbres (ramifiés au maximum jusqu'à l'ordre 5) sauf sur les 5 arbres du dernier prélèvement pour lesquels seuls les axes d'ordre 1, 2 et 3 ont été décrits. Quelques axes (moins de 1\%) ont été cassés lors de l'excavation, si bien que certaines données de longueur sont manquantes. D'autres axes ont été bloqués dans leur croissance par des amas de grains de perlite. Les axes d'ordre 1 et 2 dans cette situation, repérables à la forme de leur partie apicale (nettement épaissie ou en crosse), ont été identifiés. Les racines n'ont pas atteint les parois du conteneur, sauf pour le dernier prélèvement où certains pivots ont touché le fond.

\section{MODÉLISATION ET ESTIMATION}

\section{Croissance des axes de différents ordres}

En rhizotrons, dans des conditions voisines de culture et sur la même durée, on observe des courbes de croissance à vitesse accélérée, cons- tante, ou ralentie (Pagès, données non publiées). Pour décrire la croissance des axes de différents ordres, nous avons recherché un modèle permettant de représenter ces formes générales. Nous avons supposé que la forme des courbes de croissance de tous les axes d'un ordre de ramification donné est la même : les courbes de croissance de toutes les racines d'un ordre donné sont affines. La variabilité de vigueur au sein d'un ordre est traduite uniquement par la variabilité du coefficient de l'affinité. Ce coefficient détermine à la fois les rapports entre les longueurs et entre les vitesses de croissance des racines d'un ordre donné, à âge égal. Au sein d'un ordre de ramification donné, on observe fréquemment une distribution dissymétrique des courbes de croissance : peu de racines s'allongent fortement alors que de nombreuses racines s'allongent très peu. Nous avons donc choisi un modèle où la distribution du coefficient de l'affinité est dissymétrique.

Pour décrire la croissance d'un axe i d'ordre n, nous avons retenu le modèle suivant :

$$
L_{i}^{(n)}=\beta^{(n)}\left(T-t_{i}-t^{(n)}\right)^{\alpha(n)} e^{v_{i}}
$$

dans lequel :

$\mathrm{L}_{\mathrm{i}}{ }^{(\mathrm{n})}$ est la longueur à la date $\mathrm{T}\left(\mathrm{T}>\mathrm{t}_{\mathrm{i}}+\mathrm{t}^{(\mathrm{n})}\right)$,

$t_{i}$ est la date de formation du fragment de racine qui supporte l'axe $i$ (pour les axes d'ordre $1, t_{i}$ vaut 0 ),

$\mathrm{t}^{(\mathrm{n})}$ est le temps écoulé avant que le fragment de racine support puisse se ramifier,

$\alpha^{(\mathrm{n})}$ est un paramètre qui définit la concavité de la courbe $\left(\alpha^{(n)}>0\right)$,

$\beta^{(n)} \mathrm{e}^{v_{i}}$ est le coefficient de l'affinité entre courbes de croissance d'axes d'un même ordre.

Ce coefficient aura une distribution dissymétrique, conformément aux observations, si nous faisons l'hypothèse d'une distribution normale du logarithme de ce coefficient, d'où la formulation choisie pour ce coefficient. Étant donnée la signification biologique de ce coefficient, nous appellerons désormais $\log \left(\beta^{(n)}\right)+v_{i}$ la vigueur de l'axe $i, \log (\beta(n))$ étant la vigueur moyenne d'un axe d'ordre $n$ et $v_{i}$ la différence entre la vigueur de l'axe $i$ et la vigueur moyenne. Les $v_{i}$ sont distribués selon une loi normale d'espérance nulle et de variance $\sigma^{(n) 2} . L_{i}{ }^{(n)}$ suit donc une loi lognormale à une date donnée. Pour chaque ordre décrit, les paramètres sont donc: $\kappa^{(n)}, \alpha^{(n)}, \beta^{(n)}$, $\sigma^{(n)}$.

Dans ce modèle, la vigueur de la racine est une caractéristique de chaque axe. Les autres 
paramètres étant connus, elle détermine totalement sa courbe de croissance.

Les paramètres estimés figurent dans le tableau I. La figure 2 représente les nuages de longueur des racines en fonction du temps, ainsi que les courbes de croissance estimées, avec des bandes de confiance individuelles à $5 \%$. Ces premiers résultats montrent que le temps écoulé avant que le fragment de racine support puisse se ramifier (paramètre $\left.t^{(n)}\right)$ augmente avec l'ordre de ramification. Les valeurs de $\alpha^{(n)}$ ont tendance à décroître d'un ordre au suivant : de 1,46 pour l'ordre 1 (croissance accélérée) jusqu'à 0,41 pour l'ordre 4 (croissance amortie). Les vitesses de croissance moyennes montrent également une tendance générale à la décroissance d'un ordre au suivant (sur les 30 premiers j de croissance, on peut calculer des vitesses de croissance moyennes de $7 \mathrm{~mm} / \mathrm{j}$ pour l'ordre 1, 2 $\mathrm{mm} / \mathrm{j}$ pour l'ordre 2, $0,5 \mathrm{~mm} / \mathrm{j}$ pour l'ordre 3 et 0,3 $\mathrm{mm} / \mathrm{j}$ pour l'ordre 4). La variabilité des vitesses de croissance est relativement faible sur l'ordre 1 , alors qu'elle est très importante sur les ordres 2,3 et 4 . La normalité des résidus, donc la normalité de la distribution des vigueurs telles que définies précédemment, est relativement bien vérifiée, surtout sur les ordres 2,3 et 4 .
Tableau I. Estimations des paramètres des courbes de croissance.
$\mathrm{t}(\mathrm{n})$
$\alpha^{(n)}$
$\beta^{(n)}$
$\sigma^{(n)}$

\begin{tabular}{lllll} 
ordre 1 & 2,01 & 1,46 & 1,55 & 0,19 \\
ordre 2 & 2,14 & 0,89 & 2,70 & 0,86 \\
ordre 3 & 2,39 & 0,71 & 1,33 & 0,78 \\
ordre 4 & 6,80 & 0,41 & 2,07 & 0,70 \\
\hline
\end{tabular}

\section{Ramification des axes}

Pour étudier la ramification des axes, nous avons admis que celle-ci se produit de façon strictement acropète. Le caractère acropète de l'apparition de la ramification est en effet une des constantes de la morphogenèse racinaire chez de nombreuses espèces (Klepper, 1987). Il a été montré pour le système racinaire juvénile de plusieurs espèces ligneuses (Colin-Belgrand et al, 1989) et en particulier chez le jeune pêcher (Pagès et al, 1991). Cela revient à supposer qu'un fragment élémentaire de racine ne peut se ramifier qu'après un temps de latence donné et pendant un temps court. De ce fait, on peut re-

\section{ORDRE 1}

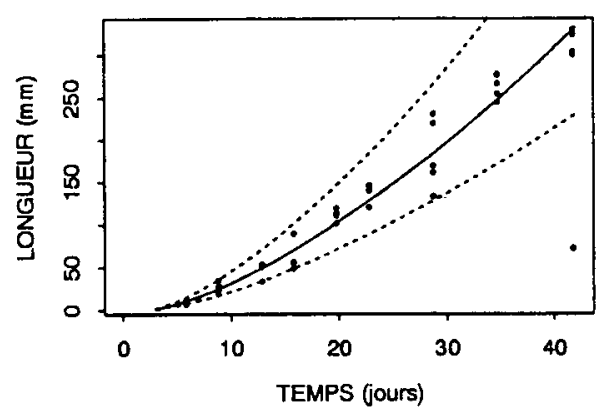

ORDRE 3

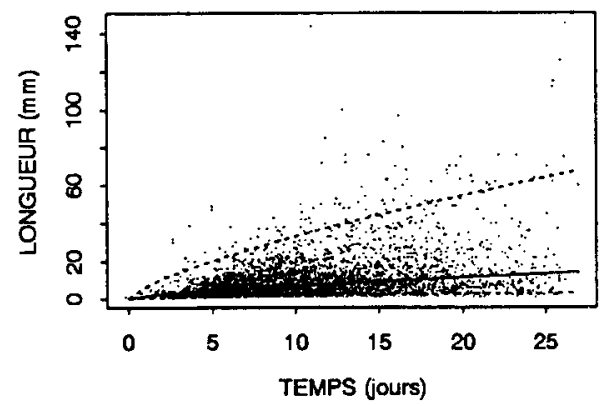

ORDRE 2

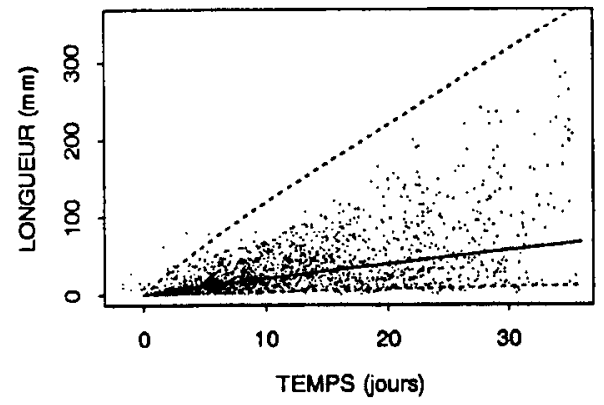

ORDRE 4

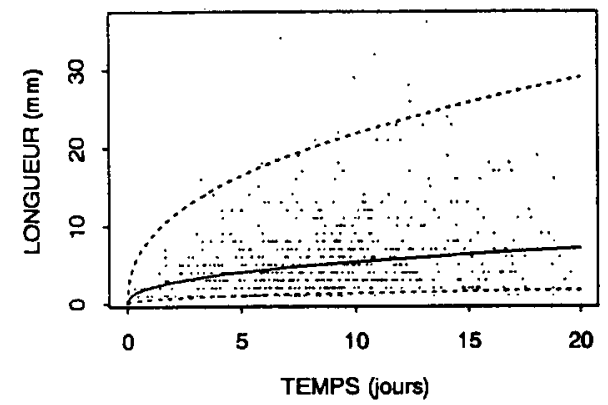

Fig 2. Longueur des racines fonction du temps. Valeurs observées ( $\bullet$ ), courbes moyenns estimées (en traits pleins) et enveloppes de confiance à $95 \%$ (en traits pointillés). 
garder la ramification comme un processus temporel, en utilisant l'équation (1) pour estimer les dates auxquelles se sont produites les différentes ramifications.

Différentes statistiques ont été estimées sur les processus de ramification. L'étude de leur intensité (c'est-à-dire du nombre moyen de ramifications apparaissant sur un axe par unité de temps) en fonction du temps, de la durée écoulée entre une date donnée quelconque et la première ramification postérieure à cette date, de la durée moyenne écoulée entre une ramification et la suivante n'a pas conduit à rejeter l'hypothèse d'un processus stationnaire (Diggle, 1983).

De plus, il n'est pas apparu de liaison entre les durées séparant des ramifications successives. Enfin, les histogrammes de ces durées (fig 3) n'ont pas conduit à rejeter l'hypothèse d'un processus de Poisson. Un tel processus se traduit par l'apparition totalement aléatoire, avec une probabilité $\lambda \mathrm{dt}$, d'une ramification sur le segment
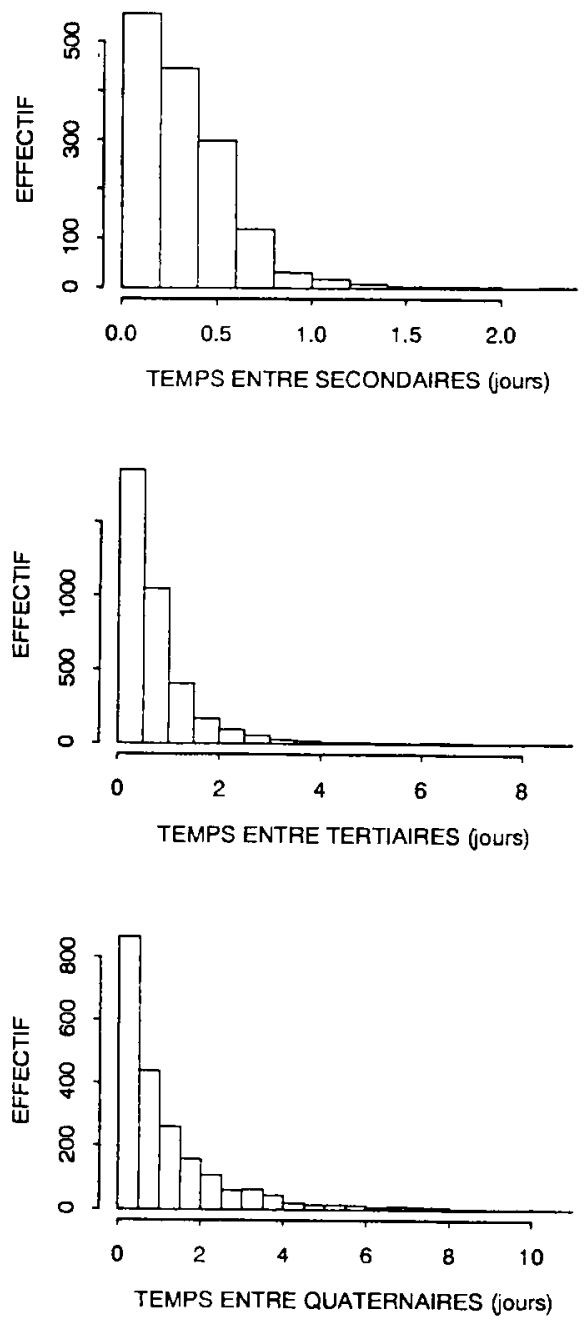

Fig 3. Histogrammes de répartition des durées séparant deux racines successives. de longueur $\mathrm{d} L$, ramifiable pendant l'intervalle de temps $[t, t+\mathrm{d} t]$. Si la racine porteuse croît avec un paramètre $\alpha^{(n)}>1$, alors la ramification vue comme un processus spatial sera de plus en plus espacée; si $\alpha^{(n)}<1$, elle sera de plus en plus rapprochée.

Pour chaque racine, l'intensité de ramification $\lambda$ est estimé par $\lambda=N / T$ où $N$ est le nombre de ses ramifications et $T$ est le temps pendant lequel elle a pu se ramifier, donné par $T=t^{\prime}-t$ avec $t^{\prime}$ : durée écoulée depuis l'apparition de la racine, $t$ temps nécessaire à l'apparition de la première ramification sur cette racine.

L'étude de la distribution des intensités de ramification estimées pour chaque racine montre une importante variabilité de ces intensités au sein de chaque ordre (fig 4, traits pleins). Quel que soit l'ordre, ces intensités sont comprises entre 0 et 5 racines/jour. La densité de l'intensité de l'ordre 1 semble unimodale, mais le nombre de points de mesure est trop faible pour l'affir-
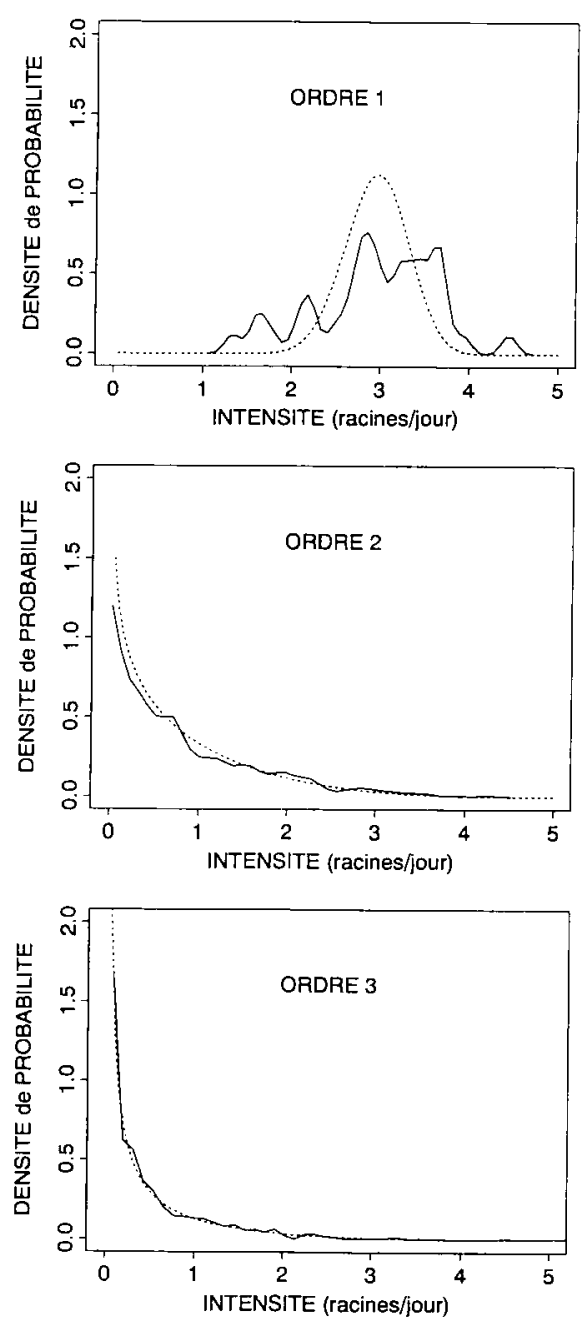

Fig 4. Courbes de densité de probabilité des intensités de ramification observées (en traits pleins) et ajustées (en traits pointillés). 
mer. La densité des intensités des ordres 2 et 3 est continuellement décroissante, ce qui correspond à un nombre important de racines à faible intensité de ramification.

Les distributions de ces intensités ont été modélisées pour chaque ordre par une loi Bêta de paramètres $a$ et $b$, sur l'intervalle $[0,5]$ et ajustées par la méthode des moments (Kendall et al, 1979; Borovkov, 1987).

Les ajustements obtenus sont donnés par les courbes pointillées de la figure 4 . Si l'on excepte le premier ordre, pour lequel on dispose de relativement peu de points pour estimer la densité empirique, ce qui explique l'allure relativement chaotique de la courbe, les ajustements des courbes sont relativement bons. Les valeurs correspondantes des paramètres de la loi Bêta sont données dans le tableau II.

Nous pouvons noter la décroissance des valeurs des paramètres estimés d'un ordre au suivant, à laquelle correspond une décroissance de la moyenne et de la variance de l'intensité $\lambda(n)$.

Remarque :

La modélisation mise en œuvre est caractérisée par l'imbrication des modèles de croissance et de ramification et des différents ordres. De ce fait, une estimation globale du modèle aurait été extrêmement lourde. Nous avons par conséquent effectué des estimations conditionnelles aux ordres précédents (par moindres carrés). Par conséquent la qualité des estimations des paramètres d'un ordre donné dépend de celle des ordres précédents.

\section{VALIDATION}

\section{Densité spatiale de ramification}

Les processus de croissance et de ramification qui sont à la base du modèle proposé sont des processus temporels. Cependant, il est possible de faire le lien avec les variables d'état décrivant des caractéristiques spatiales, concernant notamment les positions des racines le long des axes porteurs. Ainsi, la position des racines d'ordre $i+$ 1 le long d'une racine $\imath$ sachant sa vigueur $v_{i}$ et son intensité temporelle de ramification $\lambda_{i}$ est décrite comme un processus de Poisson spatial inhomogène. Rappelons que l'intensité temporelle correspond au nombre moyen de racines d'ordre $i$ + 1 apparaissant sur une racine d'ordre $i$ par unité de temps, l'intensité spatiale au nombre moyen
Tableau II. Estimation des paramètres des lois Bêta décrivant l'intensité du processus de ramification pour chacun des ordres.

$a \quad b$

$\begin{array}{lrr}\text { ordre 1 } & 28,66 & 19,90 \\ \text { ordre 2 } & 0,71 & 4,03 \\ \text { ordre 3 } & 0,23 & 3,69\end{array}$

de racines d'ordre $i+1$ apparaissant sur une racine d'ordre $i$ par unité de longueur. L'intensité spatiale de racines de paramètres $\alpha$ et $\beta$, de vigueur $v_{i}$ et d'intensité temporelle de ramification $\lambda_{i}$, est donnée par la formule :

$$
\gamma\left(\mathrm{d} / \mathrm{v}_{\mathrm{i}}, \lambda_{\mathrm{i}}\right)=\frac{\lambda_{\mathrm{i}} \beta^{-1 / \alpha} \mathrm{e}^{\mathrm{v}_{\mathrm{i}} / \alpha}}{\alpha \mathrm{d}^{(\alpha-1) / \alpha}}
$$

où $d$ est la distance depuis la base de la racine. Par souci de concision, l'exposant $(n)$ des paramètres $\alpha^{(n)}$ et $\beta^{(n)}$ a été supprimé.

L'intensité moyenne de ramification sur une racine est alors :

$$
\mathrm{E}(\gamma(\mathrm{d}))=\frac{\mathrm{m} \beta^{-1 / \alpha} \mathrm{e}^{\sigma^{2} /\left(2 \alpha^{2}\right)}}{\alpha \mathrm{d}^{(\alpha-1) / \alpha}}
$$

son moment d'ordre 2

$$
E\left(\gamma(d)^{2}\right)=\frac{M \beta^{-2 / \alpha} \mathrm{e}^{2 \sigma^{2} / \alpha^{2}}}{\alpha^{2} \mathrm{~d}^{2(\alpha-1) / \alpha}}
$$

d'où la variance de l'intensité du processus spatial de ramification $\operatorname{var}(\gamma(\mathrm{d}))=\mathrm{E}\left(\gamma(\mathrm{d})^{2}\right)-E\left(\chi_{(d))^{2}}\right.$, avec dans les formules précédentes,

$$
m=5 \frac{a}{a+b}
$$

l'intensité moyenne de ramification dans le temps et

$$
M=25 \frac{a b}{(a+b+1)(a+b)^{2}}+m^{2}
$$

son moment d'ordre 2.

Nous avons présenté (fig 5) les intensités de ramification observées, les intensités moyennes 
simulées en fonction de la distance depuis la base, pour les racines d'ordre 1,2 et 3 et les écarts types de ces intensités moyennes simulées. Les effectifs observés correspondent, pour chaque tranche de position, aux nombres de ramifications observées dans la tranche de position considérée, rapportés aux nombres de racines susceptibles d'être porteuses, vu leur âge et leur vigueur. Sur l'ordre 1, il y a une bonne concordance entre valeurs simulées et valeurs observées, et on retrouve bien le fait que les racines secondaires sont de plus en plus espacées le long des pivots au niveau de la forme des courbes d'intensité moyenne simulées en fonction de la distance depuis la base, ce qui était at-

\section{SUR L'ORDRE 1}
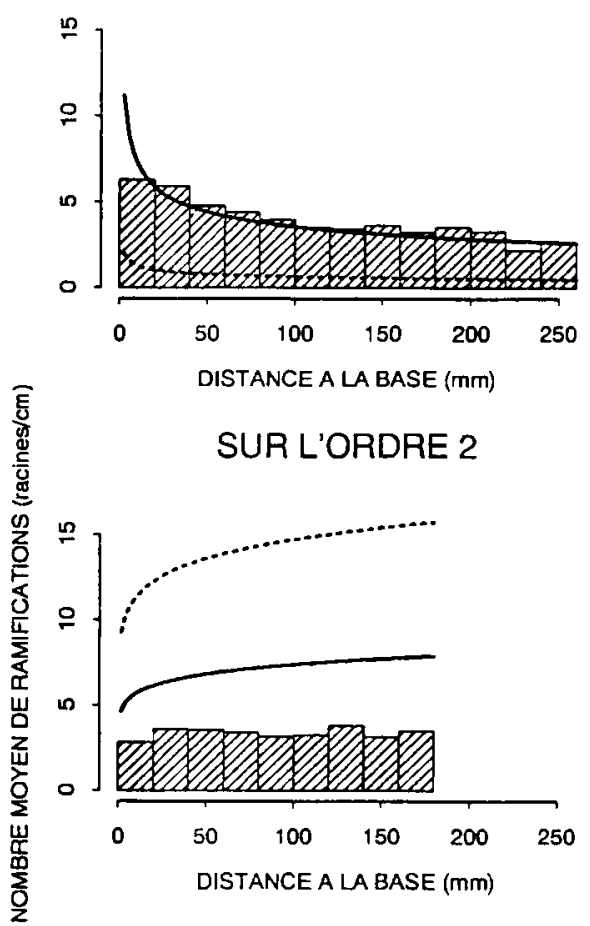

SUR L'ORDRE 3

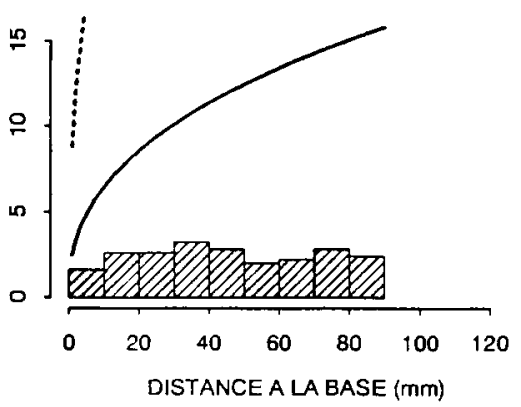

Fig 5. Intensités de ramification en fonction de la distance à la base : intensités observées (diagrammes en bâtons), moyennes (en traits pleins) et écart types (en traits pointillés) des intensités simulées. tendu compte tenu de la forme des courbes de croissance à l'ordre 1.

Sur les ordres 2 et 3 , par contre, les moyennes des valeurs simulées s'écartent beaucoup plus des données observées mais les variabilités associées sont elles aussi très importantes. L'importance de ces variabilités est due principalement à la forte variabilité de vigueur pour ces ordres, comme le montre l'étude de l'intensité de ramification conditionnelle à la vigueur, $E\left(\gamma\left(d / v_{i},\right)\right)$. Nous avons tracé les valeurs de $\mathrm{E}\left(\gamma\left(\mathrm{d} / \mathrm{v}_{\mathrm{i}},.\right)\right)$ pour une vigueur médiane et pour des vigueurs correspondant aux quantiles ${ }^{\star} 5 \%$ et $95 \%$ (fig 6). On note sur ce graphique que les valeurs observées sur la figure 5 sont proches

SUR L'ORDRE 1
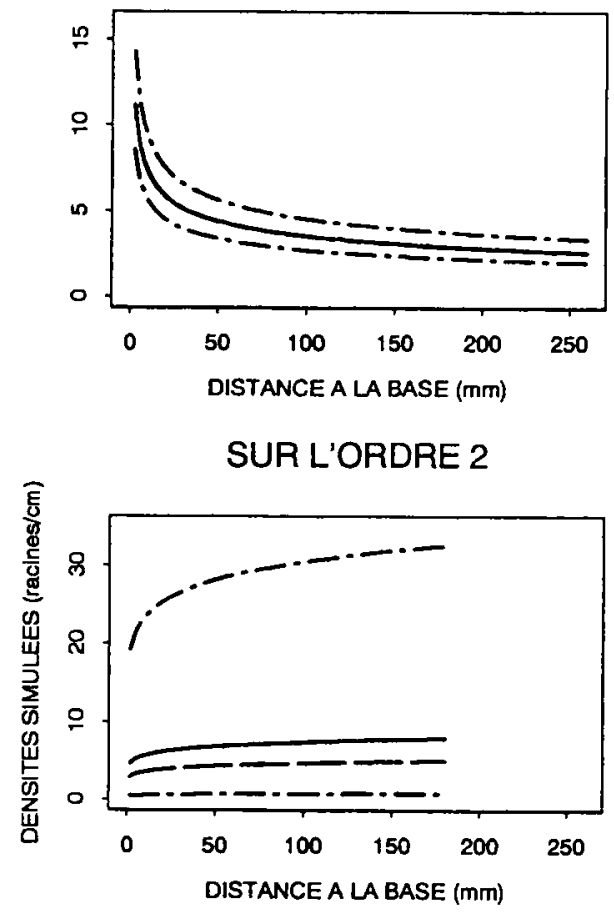

SUR L'ORDRE 3

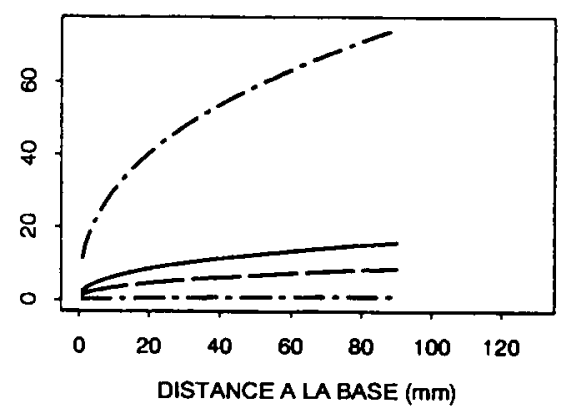

Fig 6. Intensités de ramification simulées conditionellement à la vigueur, en fonction de la distance à la base: courbes pour des valeurs de vigueur correspondant à la médiane ($\rightarrow$ ) et aux quantiles $5 \%$ et $95 \%(-\longrightarrow$ - $\longrightarrow$ replacées par rapport à la courbe de l'intensité moyenne (en traits pleins).

* $\mathrm{q}$ est le quantile $\mathrm{x} \%$ d'une variable aléatoire si $\mathrm{x} \%$ des individus de la population ont une valeur inférieure ou égale à $\mathrm{q}$. 
des courbes correspondant aux vigueurs médianes. Les courbes correspondant au quantile $95 \%$ s'éloignent plus des valeurs observées que les courbes à $5 \%$. L'écart observé entre courbes moyennes théoriques et observées est donc dû principalement aux valeurs extrêmes de la vigueur et dont l'exponentielle amplifie les effets. Ceci montre la forte influence, au niveau de la modélisation de la ramification, du choix de la loi normale pour modéliser la variabilité de la vigueur de croissance des racines.

II faut, en outre, souligner que les densités observées à une distance élevée de la base ne sont en fait observées que sur quelques racines de vigueur particulièrement forte, notamment pour les ordres 2 et 3 . On peut donc s'attendre à des écarts entre valeurs simulées moyennes et valeurs observées, avec des valeurs observées plus faibles.

\section{Confrontation des cinétiques globales}

Ce modèle a été élaboré à partir d'hypothèses faites sur les racines considérées individuellement, et estimé sur des racines considérées indépendantes. Aussi nous a-t-il semblé intéressant de confronter ses résultats aux cinétiques globales observées sur les systèmes racinaires

\section{ORDRE 1}

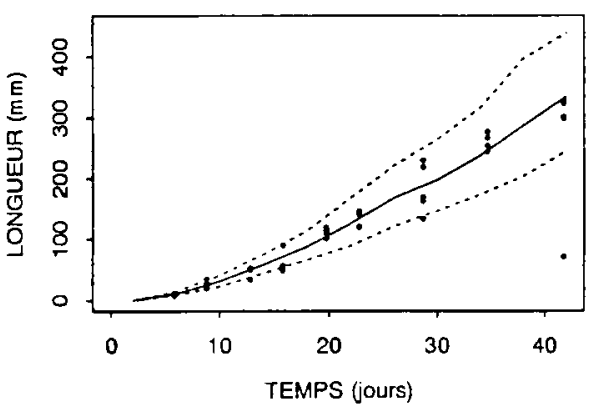

ORDRE 3

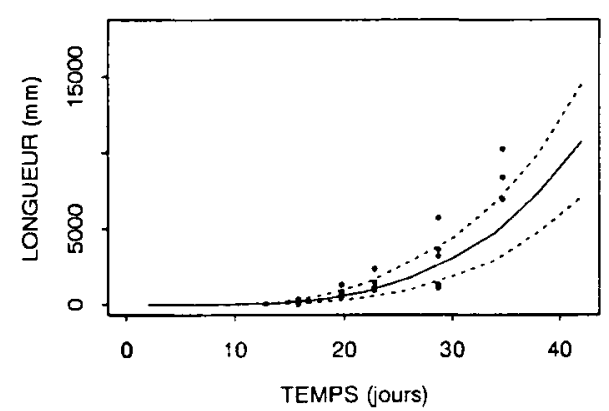

en nombres de racines et longueurs cumulées pour les différents ordres (figs 7 et 8 ). Ces variables représentent en effet en partie l'absorption potentielle du système racinaire car elles décrivent la surface d'échange offerte par la plante au sol, surface pratiquement déterminée à partir de la connaissance de la longueur des différents ordres de ramification. La variable longueur a d'ailleurs été largement utilisée dans les études de fonctionnement du système racinaire (Böhm, 1979; De Willingen et Van Noordwijk, 1987). Le nombre de racines pour chacun des ordres indique comment cette longueur est segmentée (ou structurée) au cours du temps, et quantifie le nombre d'apex. Ces derniers peuvent présenter un fonctionnement particulier au sein du système racinaire (revue de Lucas, 1987 et celle de Habib et al, 1988).

Les simulations présentées sur les figures 7 et 8 montrent que le modèle décrit convenablement l'ensemble de ces cinétiques, aussi bien en moyenne qu'en variabilité. Comme les courbes en pointillés représentent les limites de confiance à 5 et $95 \%$, et que le nombre d'individus sur lesquels nous disposons de mesures varie de 38 à 45 , nous pouvons nous attendre à avoir 4 à 5 individus en dehors de ces limites Ces nombres sont proches de ceux que l'on obtient effectivement, et on observe en général une bonne des-

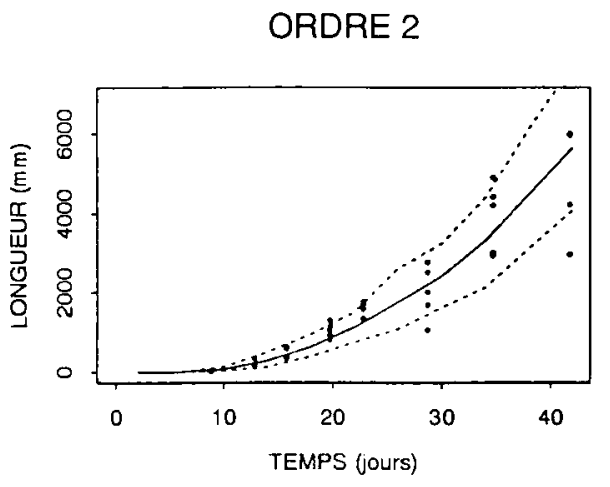

ORDRE 4

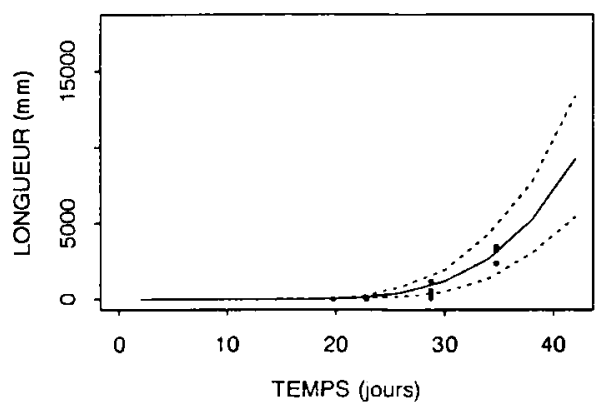

Fig 7. Longueur totale des racines d'un ordre donné en fonction du temps. Valeurs observées ( $\bullet$ ), courbe moyenne simulée (en traits pleins) et enveloppe de confiance à $95 \%$ (en traits pointillés). 
ORDRE 2

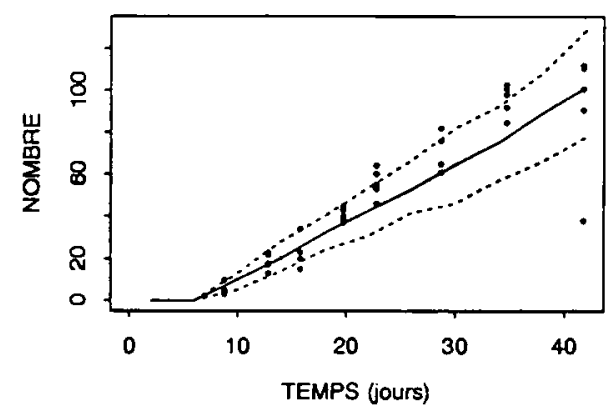

ORDRE 4

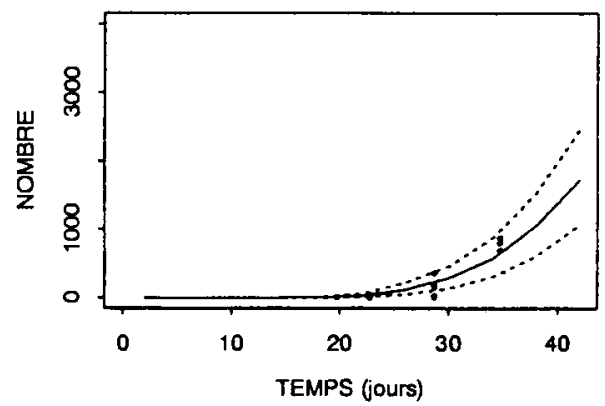

ORDRE 3

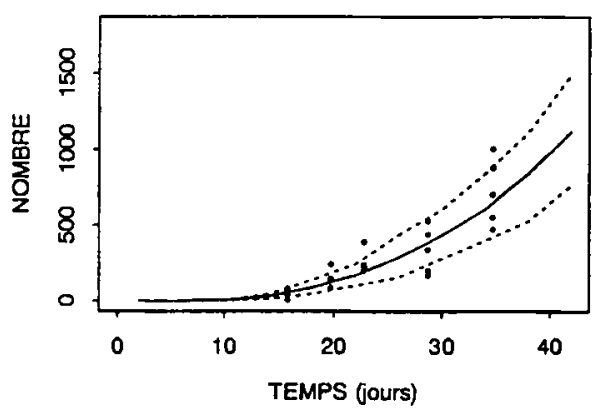

Fig 8. Nombre total de racines d'un ordre donné en fonction du temps. Valeurs observées (๑), courbe moyenne simulée (en traits pleins) et enveloppe de confiance à $95 \%$ (en traits pointillés).

cription de l'évolution de la variabilité au cours du temps. Globalement, le modèle n'est pas rejeté au vu de ces résultats.

On peut se demander toutefois, au vu de la courbe d'évolution du nombre de racines d'ordre 2 , si celui-ci n'évolue pas selon une courbe de type sigmoïde plutôt que suivant une droite, ce qui est attendu dans l'hypothèse d'une intensité temporelle de ramification constante.

Avant de proposer ce modèle où la densité de ramification $\lambda^{(n)}$ est tirée selon une loi Bêta, nous avions envisagé le modèle plus simple où ce paramètre est constant à chaque ordre. Ce sousmodèle a été rejeté dans la mesure où il sousestime notablement la variabilité des nombres et des longueurs aux ordres 2,3 et 4 . Ces résultats montrent encore la nécessité de prendre en compte conjointement la variabilité de la croissance et de la ramification des racines.

\section{DISCUSSION-CONCLUSION}

Le modèle a permis, avec un nombre relativement faible de paramètres, de décrire convenablement les cinétiques de croissance et de ramifi- cation des 4 ordres racinaires, aussi bien en moyenne qu'en variabilité. Lors de ces estimations, nous avons vu la nécessité de prendre en compte à la fois la variabilité des courbes de croissance et des vitesses de ramification des différentes racines. La seule prise en compte de la variabilité sur les courbes de croissance tend en effet à sous-estimer la variance des variables d'état que nous avons étudiées.

Cette étude a en outre permis de mettre en évidence le problème du choix des modèles décrivant la variabilité observée, les queues de distribution souvent négligées au niveau de l'analyse, pouvant avoir des effets relativement importants par la suite au niveau global.

D'un point de vue statistique, ce type de données pose des problèmes d'estimation spécifiques. D'une part, l'existence de structures imbriquées ne rend pas possible dans la pratique une estimation globale des paramètres. De ce fait, les paramètres de chaque ordre ont été estimés conditionnellement aux estimations des paramètres des ordres précédents. D'autre part, le modèle de croissance proposé, simple tout en permettant de prendre en compte l'ensemble des courbes de croissance observées pour les 
ordres 1 à 4 , pose malgré tout des problèmes de fortes liaisons entre paramètres estimés. À cause de ces liaisons, il n'a pas été possible d'estimer la structure de variance-covariance des paramètres. Ces problèmes peuvent être résolus d'une part au moyen de mesures différentes comme des mesures dynamiques dans le temps permettant alors d'analyser séparément chaque ordre, d'autre part en assurant un nombre plus important de mesures au niveau du premier ordre.

Même si l'estimation des paramètres est peu précise, en raison notamment de l'«empilement" des erreurs d'un ordre de ramification au suivant, on peut remarquer des tendances générales de variation d'un ordre au suivant. Concernant la croissance, nous avons noté une diminution globale des vitesses de croissance de l'ordre 1 à l'ordre 4, avec une évolution de la concavité des courbes de croissance. Tournée vers le haut à l'ordre 1, elle est presque inexistante sur l'ordre 2 , et se trouve tournée vers le bas et de plus en plus forte pour les ordres 3 et 4 . Cette tendance à l'évolution de la croissance rapide et indéfinie (ordre 1) vers une croissance faible et définie (ordre 4) a été décrite sur un nombre plus restreint d'ordres de ramification chez d'autres espèces, comme les chênes (Riedacker et al, 1982) ou le maïs (Varney et al, 1991). Chez le jeune pêcher, cette évolution est plus graduelle que chez les espèces précitées, ce qui aboutit à un système racinaire moins hiérarchisé. Conjointement, nous avons pu quantifier une évolution de la vitesse de ramification, qui tend, elle aussi, à diminuer en moyenne d'un ordre au suivant, jusqu'à devenir presque nulle à l'ordre 4 , et une évolution du temps de latence avant ramification qui tend à augmenter. Ces évolutions se font également très progressivement. Le caractère peu hiérarchisé des systèmes racinaires observés se retrouve dans le nombre d'ordres de ramification qui était de 5 dans notre cas, alors qu'il atteint seulement 3 , exceptionnellement 4 , chez d'autres plantes comme le maïs (Varney et al, 1991).

La très grande variabilité des croissances et ramifications des racines d'ordre 2 à 4 a été prise en compte à travers l'utilisation d'un modèle stochastique. Le déterminisme de ces variations est en effet très mal connu, et plusieurs auteurs (revue de Coutts, 1987) ont noté le caractère imprévisible (apparemment aléatoire) du potentiel de croissance d'une racine en fonction de sa position le long d'une porteuse.
D'après Coutts (1987), le potentiel de croissance d'une racine (que nous pouvons rapprocher de la "vigueur» que nous avons définie) est en grande partie déterminé précocement, au stade primordium. Ceci justifie en partie le fait d'avoir fixé la vigueur au départ pour chaque racine. Plusieurs hypothèses ont été avancées concernant la causalité de ces variations de vigueur (parmi elles: courbure de la racine mère, ralentissement de celle-ci, proximité de la partie aérienne source d'assimilats). Ces hypothèses sont encore difficiles à formaliser dans le cadre d'un modèle d'architecture suffisamment simple pour être utilisé pour prévoir la distribution spatiale des racines.

Nous avons vu que le modèle était capable de rendre compte (au moins approximativement) des variables spatiales comme la longueur de la zone apicale non ramifiée sur le pivot (résultats non présentés), et la densité de ramifications le long d'une racine. L'hypothèse du temps de latence entre la formation et la ramification d'un segment de racine est justifiée notamment par les travaux d'Abadia-Fenoll et al (1986) qui ont montré sur oignon la nécessité d'un temps de maturation minimal avant la ramification d'un tissu. L'existence d'une zone apicale non ramifiée qui s'allonge lorsque la vitesse de croissance du pivot augmente est un élément supplémentaire de validation de cette hypothèse. Le raccourcissement, voire la disparition de la zone apicale non ramifiée lorsque la racine est arrêtée par un obstacle, que nous avons pu observer lors des descriptions, vont dans le même sens.

L'hypothèse d'une intensité temporelle de ramification constante au cours du temps permet, comme nous l'avons vu, de rendre compte de la diminution de la densité de ramification de la base vers l'apex des pivots. Cette diminution de densité a également été observée chez d'autres espèces ligneuses (revue de Coutts, 1987) et chez le maiis dans certains cas (Varney et al, 1991). Avec cette hypothèse, le modèle prévoit l'augmentation vers l'apex de la densité le long d'un axe dont la vitesse de croissance diminue. Cette prévision ne nous a pas semblé toujours vérifiée. Cependant, étant donné la variabilité intrinsèque du processus de ramification, il nous est difficile d'infirmer cette hypothèse pour le moment. On peut cependant noter l'existence d'hypothèses concurrentes pour expliquer la décroissance de la densité de la base vers l'apex du pivot, comme l'éloignement progressif des sources hormono-trophiques que constituent les parties aériennes. 
En l'état, et grâce à sa simplicité, ce modèle constitue un outil pour progresser dans la description et la compréhension de la morphogenèse à l'échelle du système racinaire. II peut être utilisé à différents niveaux. II permet par exemple de caractériser chacune des racines observées par le paramètre "vigueur", et ainsi de comparer entre elles des racines de position et d'âge différents, ce qui serait impossible en l'absence de modèle si on n'a pas suivi la croissance de ces racines. II peut être utilisé pour comparer différentes situations (différents systèmes racinaires ou différentes conditions de cultures par exemple) en effectuant une estimation des paramètres dans chaque cas. II peut également être intéressant dans ce cadre d'analyser les variations conjointes des paramètres de ce modèle avec l'ordre de ramification.

Par simulation, il permet d'établir une référence à partir de laquelle on peut tester la validité de certaines hypothèses en étudiant plus en détail les écarts par rapport aux données observées.

II permet également, en faisant varier les paramètres, d'étudier l'effet de différents niveaux de variabilité (croissance et ramification) sur la variabilité globale. Ceci est intéressant car les effets de l'environnement sont souvent plus sensibles sur la variabilité des croissances ou des ramifications que sur leurs moyennes, davantage régulées par la plante.

Nous avons abordé essentiellement dans cette étude la discussion des aspects structure et développement du système racinaire. II conviendra d'étudier également la distribution spatiale des racines, distribution qui joue un rôle important dans la fonction d'absorption et qui sera évidemment très dépendante de la variabilité des croissances en longueur des axes.

\section{RÉFÉRENCES}

Abadia-Fenoll F, Casero PJ, Lloret PG, Vidal MR (1986) Development of lateral primordia in decapitated adventitious roots of Allium cepa. Ann Bot 58, 103-107

Böhm W (1979) Methods of studying root systems. Ecological Studies, Springer-Verlag, Berlin, $188 p$

Borovkov A (1987) Statistique mathématique. Edition Mir, Moscou

Colin-Belgrand $M$, Pagès $L$, Dreyer $E$, Joannes $H$ (1989) Analysis and simulation of a growing root system: application to a comparative study of several tree seedlings. Ann Sci For 46 suppl, Forest
Tree Physiology (E Dreyer et al eds) Elsevier' INRA, 305-309

Coutts MP (1987) Developmental processes in tree root systems. Can J For Res 17, 761-767

Diggle AJ (1988) ROOTMAP - a model in threedimensional coordinates of the growth and structure of fibrous root systems. Plant Soil 105, 169178

Diggle PJ (1983) Statistical analysis of spatial point patterns. Acad Press, Londres

Habib R, Dron C, Pagès L (1988) Influence de l'architecture racinaire sur l'absorption hydrominérale. $8 e$ Colloque sur les recherches fruitières, 7-8/12/88 Bordeaux, INRA-CTIFL, 105-118

Hackett C, Rose DA (1972) A model of the extension and branching of a seminal root of Barley, and its use in studying relations between root dimensions. I. The model. Aust J Biol Sci 25, 669-679

Jordan MO (1986) Mise en place du système racinaire du maïs. Définition et caractérisation au champ et en conditions contrôlées des paramètres descriptifs de la structure racinaire. Construction de l'organigramme d'un modèle cinétique. Thèse de l'université Louis Pasteur de Strasbourg, $78 p$

Kendall M, Stuart A (1979) The advanced theory of statistics. Vol 2 : Inference and relationship. Griffin \& Co Ltd, Londres

Klepper B (1987) Origin, branching and distribution of root systems. In: Root development and function (PJ Gregory, JV Lake, DA Rose) Cambridge Univ Press, 103-124

Lucas WJ (1987) Functional aspects of cells in root apices. In: Root development and function (PJ Gregory, JV Lake, DA Rose) Cambridge Univ Press, 27-52

Lungley DR (1973) The growth of root systems - A numerical computer simulation model. Plant Soil 38, 145-159

Pagès $L$, Aries $F$ (1988) SARAH : modèle de simulation de la croissance, du développement et de l'architecture des systèmes racinaires. Agronomie 8 (10), 62-68

Pagès L, Jordan MO, Picard D (1989) Simulation of the maize root system architecture. Plant Soil 119, 147-154

Pagès L, Kervella J, Chadœuf J (1991) Mise en place du système racinaire de jeunes pêchers (Prunus persica). 2e Coll Int Arbre, 10-15/09/90 Montpellier (France) (sous presse)

Passioura JB (1988) Water transport in and to roots. Annu Rev Plant Physiol 39, 245-265

Porter JR, Klepper B, Belford RK (1986) A model (WHTROOT) which synchronizes root growth and development with shoot development for winter wheat. Plant Soil 92, 133-145

Riedacker A, Dexheimer J, Tavakol R, Alaoui H (1982) Modifications expérimentales de la morphogénèse et des géotropismes dans le système racinaire de jeunes chênes. Can J Bot 60, 765-778 
Rose DA (1983) The description of the growth of root systems. Plant Soil 75, 405-415

Rouskas D, Hugard J, Jonard R, Villemur P (1980) Contribution à l'étude de la germination des graines de pêcher (Prunus persica $L$ Batsch) cultivar INRA GF 305 : effets de la benzyl-amino-purine (BAP) et des gibbérellines $\mathrm{GA} 3$ et $\mathrm{GA} 4+7$ sur la levée de dormance embryonnaire et l'absence des anomalies foliaires observées sur les plantes issues de graines non stratifiées. CR Acad Sci Paris 291, 861-864
De Wilingen $P$, Van Noordwijk M (1987) Uptake potential of non-regularly distributed roots. J Plant Nutr $10,1273-1280$

Varney GT, Canny MJ, Wang XL, McCully ME (1991) The branch roots of Zea. 1. First-order branches, their number, sizes, and division into classes. Ann Bot 67, 357-364

Yorke JS, Sagar GR (1970) Distribution of secondary root growth potential in the root system of Pisum sativum. Can J Bot 48, 699-704 